\title{
TORIC INTEGRABLE GEODESIC FLOWS IN ODD DIMENSIONS
}

\author{
Christopher R. Lee and Susan Tolman
}

\begin{abstract}
Let $Q$ be a compact, connected $n$-dimensional Riemannian manifold, and assume that the geodesic flow is toric integrable. If $n \neq 3$ is odd, or if $\pi_{1}(Q)$ is infinite, we show that the cosphere bundle of $Q$ is equivariantly contactomorphic to the cosphere bundle of the torus $\mathbb{T}^{n}$. As a consequence, $Q$ is homeomorphic to $\mathbb{T}^{n}$.
\end{abstract}

\section{Introduction}

Let $Q$ be an $n$-dimensional compact Riemannian manifold. On the punctured cotangent bundle, $T^{*} Q \backslash Q$, consider the function

$$
h(q, p)=\sqrt{g_{q}^{*}(p, p)},
$$

where $g^{*}$ denotes the dual of the Riemannian metric $g$. (Here, $(q, p)$ are local coordinates on $T^{*} Q$, with $q \in Q$ and $p \in T_{q}^{*} Q$.) The geodesic flow on $T^{*} Q \backslash Q$ is the flow of the Hamiltonian vector field of $h$ with respect to the standard symplectic form $\Omega=\sum d q_{i} \wedge d p_{i}$. The geodesic flow is toric integrable if there is an effective action of the torus $\mathbb{T}^{n}=\mathbb{R}^{n} / \mathbb{Z}^{n}$ on $T^{*} Q \backslash Q$ that commutes with dilations $(q, p) \mapsto\left(q, e^{t} p\right)$, preserves the symplectic form $\Omega$, and commutes with the geodesic flow (or, equivalently, preserves $h$ ). For example, the flat metric on the torus $\mathbb{T}^{n}$ and the round metrics on the sphere $S^{2}$, the projective plane $\mathbb{R} \mathrm{P}^{2}$, and the lens spaces $S^{3} / \mathbb{Z}_{\ell}$ are all toric integrable. The $\mathbb{T}^{n}$ action on $T^{*} \mathbb{T}^{n} \backslash \mathbb{T}^{n}$ is simply the lift of the natural action on $\mathbb{T}^{n}$. In the remaining cases, the geodesic flow induces a circle action on $T^{*} Q \backslash Q$ which commutes with the lift of the natural $\mathbb{T}^{n-1}$ action on $Q$.

In [20], Toth and Zelditch employ toric integrable geodesic flows to examine connections between the dynamics of the geodesic flow and the eigenfunctions of the Laplace operator. Motivated by this work, Lerman and Shirokova prove a conjecture of Toth and Zelditch: every toric integrable metric on a torus is flat [14]. (In contrast, not every toric integrable metric on $S^{2}$ is round [4].) In [12], Lerman extends this work to show that the only toric integrable actions on the punctured cotangent bundle of the $n$-torus and the two-sphere are the standard actions. In this paper, he poses the following question: are the examples listed above the only manifolds that admit toric integrable geodesic flows? The first author partially answered this question by proving that if $Q$ is a compact three-dimensional manifold which admits a toric integrable geodesic flow, then the cosphere bundle $S\left(T^{*} Q\right)$ is either equivariantly contactomorphic ${ }^{1}$ to $\mathbb{T}^{3} \times S^{2}$, or is homotopy equivalent to $\left(S^{3} / \mathbb{Z}_{\ell}\right) \times S^{2}$,

\footnotetext{
Received by the editors June 18, 2011.

${ }^{1}$ Theorem 1.1 in [10] only explicitly states that these manifolds are diffeomorphic, but clearly proves this stronger claim.
} 
where $\ell \geq 1$ [10, Theorem 1.1]. The main goal of this paper is to determine which odd-dimensional compact manifolds admit toric integrable geodesic flows.

Our approach to toric integrable geodesic flows will be from the perspective of contact geometry. A contact form on a $(2 n-1)$-dimensional manifold $M$ is a oneform $\alpha$ such that $\alpha \wedge(d \alpha)^{n-1}$ is nowhere zero. A (co-orientable) contact structure on $M$ is a codimension one subbundle $\xi$ of $T M$ such that $\xi=\operatorname{ker} \alpha$ for some contact form $\alpha$. A contact toric manifold is a $(2 n-1)$-dimensional manifold $M$, a contact structure $\xi$ on $M$, and an effective action of the $n$-torus that preserves $\xi$.

For any Riemannian manifold $Q$, the restriction of the Liouville one-form $\alpha=$ $\sum p_{i} d q_{i}$ to the cosphere bundle $S\left(T^{*} Q\right)$ is a contact form. If $Q$ admits a toric integrable geodesic flow, then the $\mathbb{T}^{n}$-action on $T^{*} Q \backslash Q$ preserves both $S\left(T^{*} Q\right)$ and $\alpha$; hence, its cosphere bundle is a contact toric manifold. By exploiting the classification of contact toric manifolds due to Lerman, we are able to show that many contact toric manifolds cannot arise as cosphere bundles - they have the wrong cohomology.

Theorem 1.1. Let $Q$ be a compact, connected $n$-dimensional Riemannian manifold, and assume that the geodesic flow is toric integrable. If $n \neq 3$ is odd, or if $\pi_{1}(Q)$ is infinite, then the cosphere bundle of $Q$ is equivariantly contactomorphic to $\mathbb{T}^{n} \times S^{n-1}$, the cosphere bundle of $\mathbb{T}^{n}$.

Proof. To begin, we may assume that $n \neq 3$ because otherwise the claim follows immediately from [10, Theorem 1.1]; (see footnote 1). Similarly, we may assume that $n>1$.

Since the geodesic flow on $Q$ is toric integrable, the cosphere bundle $S\left(T^{*} Q\right)$ is a $(2 n-1)$-dimensional contact toric manifold.

Assume first that $\pi_{1}(Q)$ is infinite. By the homotopy long exact sequence for the bundle $S^{n-1} \rightarrow S\left(T^{*} Q\right) \rightarrow Q$, this implies that $\pi_{1}\left(S\left(T^{*}(Q)\right)=\pi_{1}(Q)\right.$ is infinite as well. Hence, if $n>3$, then by the classification of contact toric manifolds described in Theorem 2.1, $S\left(T^{*} Q\right)$ is equivariantly contactomorphic to $\mathbb{T}^{k} \times S^{2 n-k-1}$ for some $0<k \leq n$. Finally, by Proposition 4.1, this is impossible unless $k=n$. Similarly, if $n=2$, then by the classification of contact toric three-manifolds described in parts (i) and (ii) of [12, Theorem 2.18], $S\left(T^{*} Q\right)$ is homeomorphic to either $\mathbb{T}^{3}$ or $S^{1} \times S^{2}$. By Propositions 4.1 and 4.3, this is only possible if $Q$ is homeomorphic to $\mathbb{T}^{2}$. Hence, the claim follows immediately from [12, Theorem 1.3].

So assume instead that $\pi_{1}(Q)$ is finite and that $n>3$ is odd. If $\widetilde{Q}$ is the universal cover of $Q$, then $\pi_{1}(\widetilde{Q})$ and $\pi_{1}\left(S\left(T^{*} \widetilde{Q}\right)\right)$ are trivial. Moreover, $S\left(T^{*} \widetilde{Q}\right)$ is a finite-sheeted cover of $S\left(T^{*} Q\right)$, and so Lemma 2.1 implies that $S\left(T^{*} \widetilde{Q}\right)$ is a contact toric manifold. Therefore, by Theorem 2.1, $S\left(T^{*} \widetilde{Q}\right)$ can be given the structure of a principal circle bundle with curvature $\omega$ over a symplectic toric orbifold $(N, \omega)$. Since $\widetilde{Q}$ is oriented, this contradicts Proposition 4.2.

Corollary 1.1. Let $Q$ be a compact, connected n-dimensional Riemannian manifold, and assume that the geodesic flow is toric integrable. If $n \neq 3$ is odd, or if $\pi_{1}(Q)$ is infinite, then $Q$ is homeomorphic to $\mathbb{T}^{n}$. If $n \leq 3$ and $\pi_{1}(Q)$ is finite, then $Q$ is either diffeomorphic to the sphere $S^{2}$, the projective plane $\mathbb{R} P^{2}$, or the lens space $S^{3} / \mathbb{Z}_{\ell}$ for some $\ell \geq 1$. 
Proof. If $n \neq 3$ is odd, or if $\pi_{1}(Q)$ is infinite, then Theorem 1.1 implies that the cosphere bundle of $Q$ is homeomorphic to $\mathbb{T}^{n} \times S^{n-1}$. By Proposition 4.3, this is only possible if $Q$ is homeomorphic to $\mathbb{T}^{n}$.

So assume that $\pi_{1}(Q)$ is finite. If $n=3$, then by Theorem 1.1 in [10], the fundamental group of the cosphere bundle $S\left(T^{*} Q\right)$ is the cyclic group $\mathbb{Z}_{\ell}$, for some $\ell \geq 1$. By Proposition 4.4, this implies that $Q$ is a lens space. If $n=2$, the claim is obvious.

Remark 1.1. Let $Q$ be a compact $n$-dimensional Riemannian manifold with $n>2$ even and $\pi_{1}(Q)$ finite. It is easy to check that Theorem 2.1 and Proposition 4.2 imply that if the odd Betti numbers $\beta_{2 i+1}(Q):=\operatorname{dim}\left(H^{2 i+1}(Q ; \mathbb{R})\right)$ do not all vanish, then the geodesic flow is not toric integrable. However, in many cases the cohomological techniques in this paper are not sufficient to prove that the cosphere bundle $S\left(T^{*} Q\right)$ is not a contact toric manifold. For example, let $Q=S^{2} \times S^{2}$ and let $M$ be the principal circle bundle over $S^{2} \times S^{2} \times S^{2}$ with first Chern class $a+b+2 c$, where $\{a, b, c\}$ is the natural basis for $H^{2}\left(S^{2} \times S^{2} \times S^{2} ; \mathbb{Z}\right)=\mathbb{Z}^{3}$. Then $M$ is a contact toric manifold and

$$
H^{i}\left(S\left(T^{*} Q ; \mathbb{Z}\right)\right)=H^{i}(M ; \mathbb{Z})= \begin{cases}\mathbb{Z}, & i=0 \text { or } 7 \\ \mathbb{Z}^{2}, & i=2 \text { or } 5 \\ \mathbb{Z}_{4}, & i=4 \\ 0, & \text { otherwise }\end{cases}
$$

In fact, we do not know if $S^{2} \times S^{2}$ admits a toric integrable geodesic flow. A fortiori, we do not know whether the list described in Corollary 1.1 includes every compact, connected Riemannian manifold with toric integrable geodesic flow.

Remark 1.2. Let $Q$ be a compact, connected $n$-dimensional Riemannian manifold, and assume that the geodesic flow is toric integrable. Even if $n \neq 3$ is odd or $\pi_{1}(Q)$ is infinite, we do not know whether $Q$ is necessarily diffeomorphic to $\mathbb{T}^{n}$. This does not follow from Corollary 1.1; for example, when $n \geq 5$ there are fake tori that are homeomorphic to $\mathbb{T}^{n}$, but not diffeomorphic (see [9]). However, Theorem 1.1 would imply that $Q$ is diffeomorphic to $\mathbb{T}^{n}$ if we could also prove the following:

If $Q$ and $Q^{\prime}$ are homeomorphic compact manifolds and $S\left(T^{*} Q\right)$ is contactomorphic to $S\left(T^{*} Q^{\prime}\right)$, then $Q$ is diffeomorphic to $Q^{\prime}$.

However, this statement seems very difficult to prove; it is closely related to many important problems in symplectic topology; cf. [1].

\section{Contact toric manifolds}

In this section, we (partially) calculate the cohomology of all compact contact toric manifolds with $\operatorname{dim}(M)>5$. This calculation relies heavily on Lerman's classification of compact contact toric manifolds [12], which builds on ideas of Banyaga and Molino, and Boyer and Galicki [2, 3].

Theorem 2.1. Let $M$ be a compact, connected, contact toric manifold with $\operatorname{dim}(M)=$ $2 n-1>5$.

(i) If $\pi_{1}(M)$ is infinite, then $M$ is equivariantly contactomorphic to $\mathbb{T}^{k} \times S^{2 n-k-1}$ for some $0<k \leq n$. 
(ii) If $\pi_{1}(M)$ is finite, then $M$ can be given the structure of a principal circle bundle with curvature $\omega$ over a symplectic toric orbifold $(N, \omega)$.

Here the contact toric structure on $\mathbb{T}^{k} \times S^{2 n-1-k}$ is induced by the restriction of

$$
\begin{gathered}
\left.\sum p_{i} d q_{i}+\frac{1}{2} \sqrt{-1} \sum\left(\bar{z}_{i} d z_{i}-z_{i} d \bar{z}_{i}\right) \in \Omega^{1}\left(T^{k} \times R^{k} \times \mathbb{C}^{n-k}\right)\right) \quad \text { to } \\
\left\{(q, p, z) \in \mathbb{T}^{k} \times \mathbb{R}^{k} \times \mathbb{C}^{n-k} \mid\|p\|^{2}+\|z\|^{4}=1\right\} .
\end{gathered}
$$

In particular, $\mathbb{T}^{n} \times S^{n-1}$ gets its contact toric structure as the cosphere bundle of $\mathbb{T}^{n}$.

Proof. This proof is adapted from the proof of Theorem 1.3 in [12].

The contact structure on $M$ is induced by a contact form $\alpha$ that is invariant under the action of $\mathbb{T}^{n}$ on $M$. For any $X \in \mathfrak{t}$, let $X_{M}$ be the corresponding vector field on $M$. The $\alpha$-moment map $\Psi_{\alpha}: M \rightarrow \mathfrak{t}^{*}$ is then defined by

$$
\left\langle\Psi_{\alpha}(p), X\right\rangle=\alpha_{p}\left(X_{M}(p)\right)
$$

for all $p$ in $M$ and $X \in \mathfrak{t}$. Let $C(M)=\left\{t \Phi_{\alpha}(M) \mid t \in[0, \infty)\right\}$ be the cone on the image of $\Psi_{\alpha}$ in $\mathfrak{t}^{*}$.

By Lerman [12, Lemma 2.12], $\Psi_{\alpha}(p) \neq 0$ for all $p \in M$. Since $n>2$, this implies that $C(M)$ is a convex polyhedral cone [11, Theorem 1.2].

Assume first that there exists $X \in \mathfrak{t}$ such that $\left\langle\Psi_{\alpha}(p), X\right\rangle>0$ for all $p \in M$. Theorem 1.1 in [13] asserts that every contact toric manifold of this type has finite fundamental group. Moreover, by Lerman and Shirokova [14, Theorem 4.3] (see also $[3]$ ), every contact toric manifold of this type can be given the structure of a principal circle bundle over a symplectic toric orbifold $(N, \omega)$. In fact, this circle bundle has curvature $\omega$. To see this, note that in their proof they show that we may choose $X$ and $\alpha$ so that the vector field $X$ generates the circle action and also so that $\left\langle\Psi_{\alpha}(p), X\right\rangle=1$. Hence, $\alpha$ is a connection one-form on the bundle $S^{1} \rightarrow M \stackrel{\pi}{\rightarrow} N$. Finally, it is easy to check that $\pi^{*}(\omega)=d \alpha$.

Next, assume that the $\mathbb{T}^{n}$ action is free. Since $n>3$, by Banyaga and Molino [2, Theorem C] (alternatively, by Lerman [12, Theorem 2.18 (3)]) $M$ is equivariantly contactomorphic to $\mathbb{T}^{n} \times S^{n-1}$, the cosphere bundle of $\mathbb{T}^{n}$. Clearly, since $n>2$, $\pi_{1}\left(T^{n} \times S^{n-1}\right)=\mathbb{Z}^{n}$.

Finally, assume that the action is not free and that there does not exist any $X \in \mathfrak{t}$ such that $\left\langle\Psi_{\alpha}(p), X\right\rangle>0$ for all $p \in M$. Lemma 4.5 in [12] implies that, since the action is not free, $C(M) \neq \mathfrak{t}^{*}$. Since $C(M)$ is a closed convex cone, this implies that the maximal linear subspace of $C(M)$ has dimension $k$, where $0<k<n$; (see Remark 2.1). Hence, [12, Theorem 2.18(4)] implies that $C(M)$ is isomorphic to the moment cone of $M^{\prime}=\mathbb{T}^{k} \times S^{2 n-1-k}$ and that $M$ is equivariantly contactomorphic to $M^{\prime}$. Finally, note that the fact that $0<k<n$ implies that $\pi_{1}\left(\mathbb{T}^{k} \times S^{2 n-1-k}\right)=\mathbb{Z}^{k}$ is infinite.

Since these are the only three possibilities, the claim follows immediately.

Remark 2.1. Let $C \subset \mathfrak{t}^{*}$ be a closed convex cone. If there does not exist $X \in \mathfrak{t}$ such that $\langle c, X\rangle>0$ for all $c \in C \backslash\{0\}$, then $C$ contains a non-trivial linear subspace. This follows by an easy inductive argument from the well-known fact that if $C \neq \mathfrak{t}^{*}$, there exists $\xi \in \mathfrak{t}$ such that $\langle c, \xi\rangle \geq 0$ for all $c \in C$. 
Given a space $X$, let $\beta_{j}(X):=\operatorname{dim} H^{j}(X ; \mathbb{R})$ be the $j$ th Betti number of $X$. The cohomology of the manifolds listed in part (i) of Theorem 2.1 are given immediately by the Künneth formula.

Proposition 2.1. Given integers $k$ and $n$ with $0<k \leq n$,

$$
\begin{aligned}
\beta_{m}\left(\mathbb{T}^{k} \times S^{2 n-k-1}\right) & =\sum_{p=0}^{m} \beta_{p}\left(\mathbb{T}^{k}\right) \beta_{m-p}\left(S^{2 n-k-1}\right) \\
& =\left(\begin{array}{c}
k \\
m
\end{array}\right)+\left(\begin{array}{c}
k \\
2 n-m-1
\end{array}\right) \text { for all } m \in \mathbb{Z},
\end{aligned}
$$

where by convention $\left(\begin{array}{c}k \\ m\end{array}\right)=0$ unless $0 \leq m \leq k$. Moreover, $H^{*}\left(\mathbb{T}^{k} \times S^{2 n-k-1} ; \mathbb{Z}\right)$ is torsion-free.

In order to analyze the Betti numbers of the second class of manifolds described in Theorem 2.1, we need to review some facts about symplectic toric orbifolds. Since they are Kähler, the Hard Lefschetz Theorem implies the following.

Theorem 2.2. Let $\left(N^{2 n-2}, \omega\right)$ be a compact symplectic toric orbifold. Then, the map

$$
\wedge[\omega]: H^{i}(N ; \mathbb{R}) \rightarrow H^{i+2}(N ; \mathbb{R})
$$

is injective for all $i<n-1$.

Our next result is a consequence of a theorem of Danilov [5], which describes the cohomology ring of symplectic toric orbifolds.

Proposition 2.2. Let $(N, \omega)$ be a compact, connected symplectic toric orbifold. As a ring, $H^{*}(N ; \mathbb{R})$ is generated by $H^{2}(N ; \mathbb{R})$; in particular, $H^{i}(N ; \mathbb{R})=0$ for $i$ odd.

Proposition 2.3. Let $M$ be a principal $S^{1}$-bundle with curvature $\omega$ over a compact symplectic toric orbifold $(N, \omega)$. Then,

$$
\beta_{i}(M)= \begin{cases}0 & \text { if } i<n \text { is odd, } \\ \beta_{i}(N)-\beta_{i-2}(N) & \text { if } i<n+1 \text { is even. }\end{cases}
$$

Proof. We may assume without loss of generality that $N$ is connected. Consider the Gysin sequence

$$
\cdots \rightarrow H^{i-2}(N ; \mathbb{R}) \stackrel{\wedge[\omega]}{\longrightarrow} H^{i}(N ; \mathbb{R}) \rightarrow H^{i}(M ; \mathbb{R}) \rightarrow H^{i-1}(N ; \mathbb{R}) \stackrel{\wedge[\omega]}{\longrightarrow} H^{i+1}(N ; \mathbb{R}) \rightarrow \cdots
$$

If $i$ is odd then $H^{i}(N ; \mathbb{R})=0$ by Proposition 2.2 , and if $i<n$ then $\wedge[\omega]: H^{i-1}(N ; \mathbb{R}) \rightarrow$ $H^{i+1}(N ; \mathbb{R})$ is injective by Theorem 2.2 . Hence, the first case follows immediately from (2.1). Similarly, if $i$ is even then $H^{i-1}(N ; \mathbb{R})=0$, and if $i<n+1$ then $\wedge[\omega]: H^{i-2}(N ; \mathbb{R}) \rightarrow H^{i}(N ; \mathbb{R})$ is injective. Hence, the second case also follows immediately from (2.1).

Our final result is that contact toric manifolds behave favorably with respect to finite-sheeted covers.

Lemma 2.1. Let $M$ be a compact, contact toric manifold. If $\widetilde{M}$ is any finite-sheeted cover of $M$, then $\widetilde{M}$ is a compact, contact toric manifold. 
Proof. Let $p: \widetilde{M} \rightarrow M$ be the covering map, and $\alpha$ an invariant contact form on $M$ such that $\operatorname{ker}(\alpha)$ is the contact structure on $M$. Then, since $p$ is a local diffeomorphism, $p^{*} \alpha \wedge d\left(p^{*} \alpha\right)^{n-1}=p^{*}\left(\alpha \wedge(d \alpha)^{n-1}\right)$ is nowhere zero, and so $p^{*} \alpha$ is a contact form on $\widetilde{M}$. Since the covering is finite, the torus action on $M$ lifts to a torus action on $\widetilde{M}$. This lifted action is effective and preserves $p^{*} \alpha$.

\section{Cosphere bundles}

In this section, we (partially) calculate the cohomology of cosphere bundles, following McCord et al. [15]. We begin with an easy consequence of the Gysin sequence.

Lemma 3.1. Let $M=S\left(T^{*} Q\right)$, where $Q$ is a connected n-dimensional manifold. If $Q$ is orientable, fix an orientation and let $e \in H^{n}(Q ; \mathbb{Z})$ be the Euler class of the bundle $S^{n-1} \rightarrow M \stackrel{p}{\rightarrow} Q$. Then

(a) $H^{i}(M ; \mathbb{Z})=H^{i}(Q ; \mathbb{Z})$, for all $i<n-1$, and

(b) $H^{n-1}(M ; \mathbb{Z})= \begin{cases}H^{n-1}(Q ; \mathbb{Z}) \oplus \mathbb{Z} & \text { if } Q \text { is orientable and } e=0 \\ H^{n-1}(Q ; \mathbb{Z}) & \text { otherwise. }\end{cases}$

Proof. Assume first that $Q$ is orientable. The Gysin sequence of the bundle $S^{n-1} \rightarrow$ $M \stackrel{p}{\rightarrow} Q$ is exact:

$\cdots \rightarrow H^{i-n}(Q ; \mathbb{Z}) \stackrel{\cup e}{\longrightarrow} H^{i}(Q ; \mathbb{Z}) \rightarrow H^{i}(M ; \mathbb{Z}) \rightarrow H^{i-n+1}(Q ; \mathbb{Z}) \stackrel{\cup e}{\longrightarrow} H^{i+1}(Q ; \mathbb{Z}) \rightarrow \cdots$

If $i<n-1$, then $H^{i-n}(Q ; \mathbb{Z})=H^{i-n+1}(Q ; \mathbb{Z})=0$. Hence, (3.1) implies claim (a). If $Q$ is compact, then $H^{n}(Q ; \mathbb{Z})=\mathbb{Z}$; if $Q$ is not compact, then $H^{n}(Q ; \mathbb{Z})=0$. In either case, $H^{0}(Q ; \mathbb{Z})=\mathbb{Z}$ and so if $e$ is not zero, then $\cup e: H^{0}(Q ; \mathbb{Z}) \rightarrow H^{n}(Q ; \mathbb{Z})$ is injective. Hence, since $H^{(n-1)-n}(Q ; \mathbb{Z})=0$ and $H^{(n-1)-n+1}(Q ; \mathbb{Z})=\mathbb{Z}$, claim (b) follows from (3.1) with $i=n-1$.

So assume instead that $Q$ is not orientable. In this case, the Gysin sequence with twisted integer coefficients is exact:

$$
\cdots \rightarrow H^{i-n}(Q ; \mathcal{Z}) \rightarrow H^{i}(Q ; \mathbb{Z}) \rightarrow H^{i}(M ; \mathbb{Z}) \rightarrow H^{i-n+1}(Q ; \mathcal{Z}) \rightarrow H^{i+1}(Q ; \mathbb{Z}) \rightarrow \cdots
$$

For any $i \leq n-1, H^{i-n}(Q ; \mathcal{Z})=H^{i-n+1}(Q ; \mathcal{Z})=0$. Hence, (3.2) implies (a) and (b).

Our main result is adapted from the statements of [15, Theorems 2.1, 2.3, and 3.1], and the proof of [15, Theorem 2.3].

Theorem 3.1 (McCord, Meyer, and Offin). Let $M=S\left(T^{*}(Q)\right)$, where $Q$ is a compact, connected $n$-dimensional manifold. Then

(i) if $Q$ is orientable, then $\beta_{i}(M)=\beta_{n-i}(M)$ for all $i \in\{2, \ldots, n-2\}$;

(ii) if $Q$ is orientable and $n>2$, then

$\beta_{n-1}(M)= \begin{cases}\beta_{1}(M)+1 & \text { if } \sum_{i=0}^{n-2}(-1)^{i} \beta_{i}(M)+(-1)^{n-1} \beta_{1}(M)+(-1)^{n}=0, \\ \beta_{1}(M) & \text { otherwise; }\end{cases}$

(iii) if $Q$ is orientable and $n=2$, then $\beta_{1}(M) \neq 1$; and

(iv) if $Q$ is not orientable, then $H^{n}(M ; \mathbb{Z})$ is not torsion-free. 
Proof. Assume first that $Q$ is orientable; fix an orientation on $Q$. Since $Q$ is compact, the Euler class of the bundle $S^{n-1} \rightarrow M \stackrel{p}{\rightarrow} Q$ is zero exactly if the Euler characteristic $\chi(Q)=\sum_{i=0}^{n}(-1)^{i} \beta_{i}(Q)$ is zero. Therefore, Lemma 3.1 implies that

$$
\begin{aligned}
& \beta_{i}(M)=\beta_{i}(Q) \text { for all } i<n-1, \quad \text { and } \\
& \beta_{n-1}(M)= \begin{cases}\beta_{n-1}(Q)+1 & \text { if } \chi(Q)=0, \\
\beta_{n-1}(Q) & \text { otherwise. }\end{cases}
\end{aligned}
$$

Moreover, by Poincaré duality

$$
\beta_{i}(Q)=\beta_{n-i}(Q) \text { for all } i .
$$

Equations (3.3) and (3.5) imply that claim (i) holds, and also that $\beta_{n-1}(Q)=\beta_{1}(M)$ if $n>2$. Hence, since $\beta_{n}(Q)=1$, claim (ii) follows from (3.4). Finally, if $n=2$, then $Q$ is a compact orientable surface of genus $g$; in particular $H^{1}(Q ; \mathbb{R})=\mathbb{R}^{2 g}$. If $g \neq 1$, then $\chi(Q) \neq 0$ and so (3.4) implies that $\beta_{1}(M)=\beta_{1}(Q)=2 g \neq 1$. On the other hand, if $g=1$, then $\chi(Q)=0$ and so (3.4) implies that $\beta_{1}(M)=\beta_{1}(Q)+1=3 \neq 1$. This completes the proof of claim (iii).

Now assume that $Q$ is not orientable; the Gysin sequence with twisted integer coefficients is exact:

$$
\cdots \rightarrow H^{0}(Q ; \mathcal{Z}) \rightarrow H^{n}(Q ; \mathbb{Z}) \rightarrow H^{n}(M ; \mathbb{Z}) \rightarrow H^{1}(Q ; \mathcal{Z}) \rightarrow \cdots
$$

Claim (iv) follows immediately from the facts that $H^{n}(Q ; \mathbb{Z})=\mathbb{Z} / 2 \mathbb{Z}$ and $H^{0}$ $(Q ; \mathcal{Z})=0$.

\section{Cosphere bundles which are contact toric manifolds}

In this section, we compare our earlier calculations of the cohomology of cosphere bundles and the cohomology of the contact toric manifolds listed Theorem 2.1. We use this to show that $\mathbb{T}^{n} \times S^{n-1}$ is the only one of these manifolds that can be given the structure of the cosphere bundle of an odd-dimensional, oriented manifold. Moreover, we show that if the cosphere bundle of a manifold $Q$ is $\mathbb{T}^{n} \times S^{n-1}$, then $Q$ is homeomorphic to $\mathbb{T}^{n}$.

Proposition 4.1. If the cosphere bundle $M=S\left(T^{*} Q\right)$ of a manifold $Q$ is homeomorphic to $\mathbb{T}^{k} \times S^{2 n-k-1}$ for some integers $k$ and $n$ with $0<k \leq n$, then $k=n$.

Proof. Assume on the contrary that $0<k<n$. Proposition 2.1 implies that

$$
\begin{gathered}
\beta_{1}\left(\mathbb{T}^{k} \times S^{2 n-k-1}\right)=k, \quad \text { and } \\
\beta_{n-1}\left(\mathbb{T}^{k} \times S^{2 n-k-1}\right)= \begin{cases}1 & \text { if } k=n-1, \\
0 & \text { if } k<n-1\end{cases}
\end{gathered}
$$

In particular $\beta_{1}\left(S^{1} \times S^{2}\right)=1$, and so $S^{1} \times S^{2}$ fails to be a cosphere bundle of an oriented manifold by Theorem 3.1(iii). Therefore, we may assume that $n>2$. Hence, Theorem 3.1(ii) implies that if $\mathbb{T}^{k} \times S^{2 n-k-1}$ is the cosphere bundle of an orientable manifold, then $\beta_{n-1}\left(\mathbb{T}^{k} \times S^{2 n-k-1}\right) \geq \beta_{1}\left(\mathbb{T}^{k} \times S^{2 n-k-1}\right)$. But this contradicts the equations above. On the other hand, Proposition 2.1 and Theorem 3.1(iv) imply that $T^{k} \times S^{2 n-k-1}$ is not the cosphere bundle of a manifold which is not orientable. 
Proposition 4.2. Assume that $M$ is a principal $S^{1}$-bundle with curvature $\omega$ over a symplectic toric orbifold $(N, \omega)$, and that $M$ is the cosphere bundle of a compact $n$-dimensional manifold $Q$ with $n>3$. If $n$ is odd, then $Q$ is not orientable; if $n$ is even, then $\beta_{2 i+1}(Q)=0$ for all $i$.

Proof. We may assume without loss of generality that $Q$ is connected; in particular,

$$
\beta_{0}(M)=1 \text {. }
$$

Moreover, by Proposition 2.3

$$
\beta_{i}(M)=0 \text { for all } i<n \text { odd. }
$$

Assume first that $n$ is odd and $Q$ is orientable. Then (4.2) and Theorem 3.1(i) imply that

$$
\beta_{i}(M)=0 \text { for all } i \in\{1, \ldots, n-2\} .
$$

Applying Theorem 3.1(ii) to (4.1) and (4.3), we see that

$$
\beta_{n-1}(M)=1 \text {. }
$$

By induction and Proposition 2.3,

$$
\beta_{i}(N)=\sum_{j=0}^{i} \beta_{j}(M) \quad \text { for } i<n+1 \text { even. }
$$

Since $n-1>2,(4.1),(4.3)-(4.5)$ together imply that $\beta_{2}(N)=1$ and $\beta_{n-1}(N)=2$. But this is impossible, since as a ring $H^{2}(N ; \mathbb{R})$ generates $H^{*}(N ; \mathbb{R})$ by Proposition 2.2.

So assume instead that $n$ is even. By Lemma 3.1(a), (4.2) implies that $\beta_{i}(Q)=0$ for all $i<n-1$ odd. If $Q$ is orientable, then $\beta_{n-1}(Q)=\beta_{1}(Q)$ by Poincaré duality. Moreover, $\beta_{1}(Q)=0$ since $1<n-1$. On the other hand, if $Q$ is not orientable, then $\beta_{n-1}(Q)=0$ by $(4.2)$ and Lemma $3.1(\mathrm{~b})$.

Proposition 4.3. If the cosphere bundle $M=S\left(T^{*} Q\right)$ of a manifold $Q$ is homeomorphic to $\mathbb{T}^{n} \times S^{n-1}$, then $Q$ is homeomorphic to $\mathbb{T}^{n}$.

Proof. Note first that Proposition 2.1 and Theorem 3.1(iv) imply that $Q$ is orientable; fix an orientation on $Q$. If $n=2$, the claim follows from the fact that the torus is the only closed surface with $\beta_{1}\left(S\left(T^{*} Q\right)\right)=\beta_{1}\left(T^{2} \times S^{1}\right)=3$. So assume that $n>2$. If $\widetilde{Q}$ is the universal cover of $Q$, then $S\left(T^{*} \widetilde{Q}\right)$ is homeomorphic to $\mathbb{R}^{n} \times S^{n-1}$. In particular,

$$
H^{i}\left(S\left(T^{*} \widetilde{Q}\right) ; \mathbb{Z}\right)= \begin{cases}\mathbb{Z} & \text { if } i=0 \text { or } n-1, \\ 0 & \text { otherwise. }\end{cases}
$$

Since $S\left(T^{*} \widetilde{Q}\right)$ is not compact, $\widetilde{Q}$ is not compact. Therefore, $H^{n}(\widetilde{Q} ; \mathbb{Z})=0 ; a$ fortiori, the Euler class of the bundle $S^{n-1} \rightarrow S\left(T^{*} \widetilde{Q}\right) \stackrel{p}{\rightarrow} \widetilde{Q}$ vanishes. By Lemma 3.1 and (4.6), this implies that $H^{i}(\widetilde{Q} ; \mathbb{Z})=0$ for all $i>0$. Since $\widetilde{Q}$ is simply connected, by the Hurewicz Theorem this implies that $\pi_{i}(\widetilde{Q})=0$ for all $i>0$. By the homotopy long exact sequence for the covering $\widetilde{Q} \rightarrow Q$, this implies that

$$
\pi_{i}(Q)=0, \quad \text { for all } i>1 \text {. }
$$


On the other hand, by the homotopy long exact sequence for the bundle $S^{n-1} \rightarrow$ $S\left(T^{*} Q\right) \rightarrow Q$, we have

$$
\pi_{1}(Q)=\pi_{1}\left(S\left(T^{*} Q\right)\right)=\pi_{1}\left(\mathbb{T}^{n} \times S^{n-1}\right)=\mathbb{Z}^{n}
$$

Thus, $Q$ is an Eilenberg-MacLane space of type $K\left(\mathbb{Z}^{n}, 1\right)$. Since Eilenberg-MacLane spaces are unique up to weak homotopy equivalence, it follows that $Q$ and $\mathbb{T}^{n}$ are weakly homotopy equivalent. Since $Q$ is a manifold, and hence a $\mathrm{CW}$ complex, this implies that $Q$ and $\mathbb{T}^{n}$ are homotopy equivalent by Whitehead's theorem.

Moreover, in [8], Hsiang and Wall prove that if a closed $n$-manifold with $n \geq 5$ is homotopy equivalent to the $n$-torus, it is homeomorphic to the $n$-torus. In [6], Freedman shows the same result holds in dimension four. Finally, assume that $n=3$. Since $Q$ is a closed manifold and $\pi_{1}(Q)=\mathbb{Z}^{3}$ cannot be written as a non-trivial free product, Perelman's proof of the Poincaré conjecture implies that $Q$ is prime $[18,19,17]$. Since $\pi_{1}(Q) \neq \mathbb{Z}$, this implies that $Q$ is irreducible. Moreover, since $H_{1}(Q, \mathbb{R}) \neq 0, Q$ contains an orientable, incompressible surface. Hence, since $Q$ is oriented, Waldhausen's theorem implies that $Q$ is homeomorphic to $T^{3}[7]$.

Proposition 4.4. If $M$ is the cosphere bundle of a compact three-manifold $Q$ and $\pi_{1}(M)=\mathbb{Z}_{\ell}$ for some $\ell \geq 1$, then $Q$ is diffeomorphic to the lens space $S^{3} / \mathbb{Z}_{\ell}$.

Proof. By the long exact sequence for the fibration $S^{2} \rightarrow S\left(T^{*} Q\right) \rightarrow Q$, the assumptions imply that $\pi_{1}(Q)=\mathbb{Z}_{\ell}$. Hence, the claim follows trivially from Thurston's elliptizaton conjecture, which states that every closed three-manifold with finite fundamental group is quotient of $S^{3}$ by a finite subgroup of $S O(4)$ acting freely. The elliptization conjecture is itself an easy consequence of Thurston's geometrization conjecture, proved by Perelman $[18,19,16]$. To see this, let $M$ be a prime threemanifold with finite fundamental group. Since $\pi_{1}(M)$ is finite, $M$ does not admit any incompressible tori or Klein bottles. Therefore, the geometrization conjecture implies that $M$ admits a locally homogeneous metric. Finally, $S^{3}$ is the only compact model geometry.

\section{References}

[1] M. Abouzaid, Framed bordism and Lagrangian embeddings of exotic spheres, (2008), 1-98, arXiv:0812.4781v1 [math.SG].

[2] A. Banyaga and P. Molino, Complete integrability in contact geometry, Penn State preprint PM 197, 1996.

[3] C. P. Boyer and K. Galicki, A note on toric contact geometry, J. Geom. Phys. 35(4) (2000), $288-298$.

[4] Y. Colin de Verdière, Spectre conjoint d'opérateurs pseudo-différentiels qui commutent. II. Le cas intégrable, Math. Z. 171(1) (1980), 51-73.

[5] V. I. Danilov, The geometry of toric varieties, Uspekhi Mat. Nauk 33(2(200)) (1978), 85-134, 247.

[6] M. H. Freedman, The disk theorem for four-dimensional manifolds, Proc. Int. Congress of Mathematicians, 1, 2 (Warsaw, 1983), 647-663, PWN, Warsaw (1984).

[7] J. Hempel, 3-Manifolds, Princeton University Press, Princeton, NJ, 1976, Ann. of Math. Studies, no. 86.

[8] W.-c. Hsiang and C. T. C. Wall, On homotopy tori. II, Bull. London Math. Soc. 1 (1969) $341-342$.

[9] M. Kreck and J. A. Schafer, Classification and stable classification of manifolds: some examples, Comment. Math. Helv. 59(1) (1984), 12-38. 
[10] C. R. Lee, Obstructions to toric integrable geodesic flows in dimension 3, Ann. Global Anal. Geom. 30(4) (2006), 397-406.

[11] E. Lerman, A convexity theorem for torus actions on contact manifolds, Illinois J. Math. 46(1) (2002), 171-184.

[12] - Contact toric manifolds, J. Symplectic Geom. 1(4) (2003), 785-828.

[13] - Homotopy groups of K-contact toric manifolds, Trans. Amer. Math. Soc. 356(10) (2004), 4075-4083 (electronic).

[14] E. Lerman and N. Shirokova, Completely integrable torus actions on symplectic cones, Math. Res. Lett. 9(1) (2002), 105-115.

[15] C. McCord, K. R. Meyer, and D. Offin, Are Hamiltonian flows geodesic flows?, Trans. Amer. Math. Soc. 355(3) (2003), 1237-1250 (electronic).

[16] J. Morgan and G. Tian, Ricci flow and the Poincaré conjecture, Vol. 3, Clay Mathematics Monographs, American Mathematical Society, Providence, RI, 2007.

[17] Completion of the Proof of the Geometrization Conjecture, (2008), 1-84, arXiv: 0809.4040v1 [math.DG].

[18] G. Perelman, The entropy formula for the Ricci flow and its geometric applications, (2002), 1-39, arXiv:math/0211159v1 [math.DG].

[19] - Ricci flow with surgery on three-manifolds, (2003), 1-22, arXiv:math/0303109v1 [math.DG].

[20] J. A. Toth and S. Zelditch, Riemannian manifolds with uniformly bounded eigenfunctions, Duke Math. J. 111(1) (2002), 97-132.

Department of Mathematics, University of Portland, 5000 N. Willamette Blvd., PortLAND, OR 97203, USA

E-mail address: leec@up.edu

Department of Mathematics, University of Illinois, 1409 W. Green St., Urbana, IL 61801, USA

E-mail address: stolman@math.uiuc.edu 